\title{
FRACTIONAL DISCRETE PROCESSES: COMPOUND AND MIXED POISSON REPRESENTATIONS
}

\author{
LUISA BEGHIN, ${ }^{*}$ Sapienza Università di Roma \\ CLAUDIO MACCI, ${ }^{* *}$ Università di Roma Tor Vergata
}

\begin{abstract}
We consider two fractional versions of a family of nonnegative integer-valued processes. We prove that their probability mass functions solve fractional Kolmogorov forward equations, and we show the overdispersion of these processes. As particular examples in this family, we can define fractional versions of some processes in the literature as the Pólya-Aeppli process, the Poisson inverse Gaussian process, and the negative binomial process. We also define and study some more general fractional versions with two fractional parameters.
\end{abstract}

Keywords: Cox process; doubly stochastic Poisson process; negative binomial process; Pólya-Aeppli process; Poisson inverse Gaussian process

2010 Mathematics Subject Classification: Primary 26A33; 33E12; 60G22

\section{Introduction}

In this paper we consider a large family of nonnegative integer-valued processes which we denote by $\{M(t): t \geq 0\}$ and defined by

$$
M(t):=\sum_{k=1}^{N_{\lambda}(t)} X_{k},
$$

where $\left\{X_{n}: n \geq 1\right\}$ is a sequence of independent and identically distributed (i.i.d.) positive and integer-valued random variables, independent of a (nonfractional) Poisson process $\left\{N_{\lambda}(t): t \geq\right.$ $0\}$ with intensity $\lambda$. Throughout this paper we also deal with a mixed Poisson representation of the process in (1); more precisely, we mean $\left\{N_{1}(S(t)): t \geq 0\right\}$, where $\{S(t): t \geq 0\}$ is a subordinator, independent of a (nonfractional) Poisson process $\left\{N_{1}(t): t \geq 0\right\}$ with intensity 1 , such that $N_{1}(S(t))$ is distributed as $M(t)$ for each fixed $t>0$.

Our aim is to present and analyze two fractional versions of the process $\{M(t): t \geq 0\}$ (see (7) and (8) at the beginning of Section 3, below). This allows us to define fractional generalizations of some processes in the literature, which include the Pólya-Aeppli process, the Poisson inverse Gaussian process, and the negative binomial process. These processes are commonly used when the empirical count data exhibit overdispersion, i.e. when the sample variance is larger than the sample mean; moreover, it is known that mixed Poisson processes provide simple counting models with overdispersion. We shall see that the fractional versions have the same feature.

Received 17 January 2013; revision received 7 March 2013.

* Postal address: Dipartimento di Scienze Statistiche, Sapienza Università di Roma, Piazzale Aldo Moro 5, I-00185 Roma, Italy. Email address: luisa.beghin@uniroma1.it

** Postal address: Dipartimento di Matematica, Università di Roma Tor Vergata, Via della Ricerca Scientifica, I-00133 Roma, Italy. Email address: macci@mat.uniroma2.it 
The two fractional versions are obtained by considering independent random time-changes of $\{M(t): t \geq 0\}$ in terms of a stable subordinator $\mathcal{A}^{\alpha}$ or its inverse $\mathcal{L}^{\alpha}$ (for $\alpha \in(0,1)$ ). This approach is inspired by the recent increasing interest on random time-changed and subordinated processes; see, for example, [7], [16], [17], [20], and [30]. These processes are widely studied and applied, mainly because their finite dimensional distributions display power law decay and thus heavy tails, either in the case of renewals and birth processes (see [19] and [24]) or in that of diffusions (see [1], [23], and [28]). We recall that fractional versions of the compound Poisson process (as the ones in this paper) have been studied in [3] and [31], in the case of continuous distributed summands.

In this paper we provide a natural fractional extension of some results in the literature. In particular, Proposition 1, below, shows that the probability mass functions of the two fractional versions of the process in (1) solve suitable Kolmogorov equations, where the classical derivatives are replaced by the fractional derivative in the Caputo sense (for the first version), and by the right-sided fractional Riemann-Liouville derivatives on $\mathbb{R}_{+}$(for the second version).

We conclude this section with an outline of the paper. We start with some preliminaries in Section 2. In Section 3 we illustrate some properties of the fractional versions of the process in (1). Some examples are presented in Section 4. Finally, Section 5 is devoted to more general fractional versions of some of the processes in Section 4 (the Pólya-Aeppli process and the Poisson inverse Gaussian process) with two fractional parameters.

\section{Preliminaries}

We start with some preliminaries on fractional calculus, i.e. we give the definitions of two fractional derivatives for real functions defined on $[0, \infty)$.

Definition 1. If $v \in(0,1)$, the Caputo derivative of order $v$ (see e.g. [11, Equation (2.4.17)] with $a=0$ ) is defined by

$$
{ }_{\mathrm{C}} D_{0+, t}^{v} f(t):=\frac{1}{\Gamma(1-v)} \int_{0}^{t}(t-s)^{-v} \frac{\mathrm{d}}{\mathrm{d} s} f(s) \mathrm{d} s \quad \text { for all } t \geq 0 .
$$

(Note that, if we set $v=1,{ }_{\mathrm{C}} D_{0+, t}^{v}$ coincides with the classical derivative $\mathrm{d} / \mathrm{d} t$; see e.g. [11, Theorem 2.1(b)]).

Definition 2. If $v \in(1, \infty)$, the right-sided fractional Riemann-Liouville derivative on $\mathbb{R}_{+}$of order $v$ (see e.g. [11, Equation (2.2.4)]) is defined by

$$
\mathrm{RL}_{-, t}^{\nu} f(t):=\frac{1}{\Gamma(m-v)}\left(-\frac{\mathrm{d}}{\mathrm{d} t}\right)^{m} \int_{t}^{\infty} \frac{f(s)}{(s-t)^{\nu-m+1}} \mathrm{~d} s \quad \text { for all } t \geq 0,
$$

where $m:=\lfloor v\rfloor+1$. (Note that, if we set $v=1,{ }_{\mathrm{RL}} D_{-, t}^{v}$ coincides with $-\mathrm{d} / \mathrm{d} t$ (i.e. the opposite of the classical derivative); see e.g. [11, Equation (2.2.5)]).

Hereafter, for simplicity, we write

$$
\frac{\mathrm{d}_{\mathrm{C}}^{v}}{\mathrm{~d} t^{\nu}}:={ }_{C} D_{0+, t}^{v} \quad \text { for } v \in(0,1), \quad \frac{\mathrm{d}_{\mathrm{RL}}^{\nu}}{\mathrm{d} t^{\nu}}:={ }_{\mathrm{RL}} D_{-, t}^{v} \quad \text { for } v \in(1, \infty) .
$$

Throughout the paper we often use the notation $Z(\cdot)$ to mean a process $\{Z(t): t \geq 0\}$. In view of what follows we recall some preliminaries on the stable subordinator $\mathcal{A}^{\alpha}(\cdot)$ of 
order $\alpha \in(0,1)$, and its inverse $\mathcal{L}^{\alpha}(\cdot)$. More precisely, let $\mathcal{A}^{\alpha}(\cdot)$ be the Lévy process (starting at the origin) such that, for each fixed $t>0$, we have

$$
\mathbb{E}\left[\mathrm{e}^{\theta \mathcal{A}^{\alpha}(t)}\right]= \begin{cases}\exp \left(-(-\theta)^{\alpha} t\right) & \text { if } \theta \leq 0 \\ \infty & \text { if } \theta>0\end{cases}
$$

thus, by referring to [29], the random variable $\mathcal{A}^{\alpha}(t)$ has a stable distribution of index $\alpha$ and parameters $\mu=0, \theta=1$, and $\sigma=(t \cos (\pi \alpha / 2))^{1 / \alpha}$. Furthermore, $\mathcal{L}^{\alpha}(\cdot)$ is defined by

$$
\mathcal{L}^{\alpha}(t):=\inf \left\{z: \mathcal{A}^{\alpha}(z)>t\right\}
$$

then we have $\mathbb{P}\left(\mathcal{L}^{\alpha}(t) \leq z\right)=\mathbb{P}\left(\mathcal{A}^{\alpha}(z) \geq t\right)$ for all $z, t>0$, and

$$
\mathbb{E}\left[\mathrm{e}^{-\theta \mathscr{L}^{\alpha}(t)}\right]=E_{\alpha, 1}\left(-\theta t^{\alpha}\right) \text { for all } \theta \geq 0 .
$$

Now we recall the definition of the two following fractional Poisson processes (see e.g. [21] for the case $v \in(0,1)$ and [25] for the case $v \in(1, \infty))$. In both cases we consider a random time-change of a nonfractional Poisson process $N_{\lambda}(\cdot)$.

1. For $v \in(0,1), N_{\lambda}^{v}(\cdot):=N_{\lambda}\left(\mathcal{L}^{v}(\cdot)\right)$, where $N_{\lambda}(\cdot)$ and $\mathcal{L}^{v}(\cdot)$ are independent.

2. For $v \in(1, \infty), \hat{N}_{\lambda}^{v}(\cdot):=N_{\lambda}\left(\mathcal{A}^{1 / \nu}(\cdot)\right)$, where $N_{\lambda}(\cdot)$ and $\mathscr{A}^{1 / \nu}(\cdot)$ are independent.

We can give some further details and we need to introduce some notation. For all integers $r \geq 0$ and for all $\gamma \in \mathbb{R}$, the rising factorial, also called the Pochhammer symbol, is defined by

$$
(\gamma)^{(r)}:= \begin{cases}\gamma(\gamma+1) \cdots(\gamma+r-1) & \text { if } r \geq 1 \\ 1 & \text { if } r=0\end{cases}
$$

the falling factorial is defined by

$$
(\gamma)_{r}:= \begin{cases}\gamma(\gamma-1) \cdots(\gamma-(r-1)) & \text { if } r \geq 1, \\ 1 & \text { if } r=0,\end{cases}
$$

and we also consider the notation

$$
\left(\begin{array}{l}
\gamma \\
r
\end{array}\right):= \begin{cases}\frac{(\gamma)_{r}}{r !}=\frac{\gamma(\gamma-1) \cdots(\gamma-r+1)}{r !} & \text { if } r \geq 1 \\
1 & \text { if } r=0\end{cases}
$$

1. If $v \in(0,1)$, it is known (see [21]) that $N_{\lambda}^{v}(t)$ is distributed as $\sum_{n \geq 1} \mathbf{1}_{\left\{T_{1}+\cdots+T_{n} \leq t\right\}}$, where $\left\{T_{n}: n \geq 1\right\}$ are i.i.d. random variables with Mittag-Leffler distribution, i.e. with continuous density

$$
f(t)=\lambda t^{\nu-1} E_{v, v}\left(-\lambda t^{\nu}\right) 1_{(0, \infty)}(t)
$$

see e.g. [4] and [18]), where $E_{\alpha, \beta}(x):=\sum_{r \geq 0}\left(x^{r} / \Gamma(\alpha r+\beta)\right)$ is the Mittag-Leffler function (see e.g. [27, p. 17]). Then, if we consider the generalized Mittag-Leffler function $E_{\alpha, \beta}^{\gamma}(x):=\sum_{r \geq 0}\left((\gamma)^{(r)} x^{r} / r ! \Gamma(\alpha r+\beta)\right.$ ), we have (see [5, Equation (2.5)])

$$
\mathbb{P}\left(N_{\lambda}^{v}(t)=k\right)=\left(\lambda t^{\nu}\right)^{k} E_{\nu, \nu k+1}^{k+1}\left(-\lambda t^{\nu}\right) \quad \text { for all integers } k \geq 0 .
$$

2. If $v \in(1, \infty)$, we have

$$
\mathbb{P}\left(\hat{N}_{\lambda}^{v}(t)=k\right)=\frac{(-1)^{k}}{k !} \sum_{r=0}^{\infty} \frac{\left(-\lambda^{1 / v} t\right)^{r}}{r !}\left(\frac{r}{v}\right)_{k} \quad \text { for all integers } k \geq 0 .
$$


We recall that this process was presented in [25] by referring to the fractional difference operator $(1-B)^{\alpha}$, for $\alpha \in(0,1]$, and it is called a space-fractional Poisson process. More precisely, the probability mass function in (5), with $v=1 / \alpha$, satisfies the following equations:

$$
\begin{gathered}
\frac{\mathrm{d}}{\mathrm{d} t} \mathbb{P}\left(\hat{N}_{\lambda}^{1 / \alpha}(t)=k\right)=-\lambda^{\alpha}(1-B)^{\alpha} \mathbb{P}\left(\hat{N}_{\lambda}^{1 / \alpha}(t)=k\right) \quad \text { for all integers } k \geq 0, \\
\mathbb{P}\left(\hat{N}_{\lambda}^{1 / \alpha}(0)=0\right)=1, \quad \mathbb{P}\left(\hat{N}_{\lambda}^{1 / \alpha}(0)=k\right)=0 \quad \text { for all integers } k \geq 1,
\end{gathered}
$$

where $B$ is the so-called backward shift operator defined by $B f(k)=f(k-1)$ and $B^{r-1} B f(k)=f(k-r)$; thus, in particular, we have

$$
(1-B)^{\alpha} f(k)=\sum_{j=0}^{\infty}(-1)^{j}\left(\begin{array}{c}
\alpha \\
j
\end{array}\right) f(k-j) .
$$

We remark that (5) here coincides with [25, Equation (1.2)], though it is written in a slightly different way.

\section{The fractional versions: compound and mixed representations}

We start with the compound representation of the fractional versions of the process $M(\cdot)$ in (1), and we refer to the processes defined in Section 2:

$$
\begin{gathered}
M^{v}(t):=M\left(\mathcal{L}^{v}(t)\right)=\sum_{k=1}^{N_{\lambda}^{v}(t)} X_{k} \quad \text { for } v \in(0,1), \\
\hat{M}^{v}(t):=M\left(\mathcal{A}^{1 / v}(t)\right)=\sum_{k=1}^{\hat{N}_{\lambda}^{v}(t)} X_{k} \quad \text { for } v \in(1, \infty) .
\end{gathered}
$$

In view of what follows, we consider the following notation for the probability mass function and the probability generating function of the random variables $\left\{X_{n}: n \geq 1\right\}$ :

$$
q_{k}:=\mathbb{P}\left(X_{1}=k\right) \quad \text { for all integers } k \geq 1, \quad g_{q}(u):=\sum_{k=1}^{\infty} u^{k} q_{k} .
$$

We also consider the notation

$$
q_{k}^{* n}:=\mathbb{P}\left(X_{1}+\cdots+X_{n}=k\right)
$$

therefore, we have $q_{k}^{* n}=0$ for all integers $k<n$.

Now we introduce the two following probability mass functions.

1. For $v \in(0,1)$, we have

$$
p_{k}^{v}(t):=\mathbb{P}\left(M_{\lambda}^{v}(t)=k\right)= \begin{cases}\mathbb{P}\left(N_{\lambda}^{v}(t)=0\right) & \text { if } k=0, \\ \sum_{n=1}^{k} q_{k}^{* n} \mathbb{P}\left(N_{\lambda}^{v}(t)=n\right) & \text { if } k \geq 1 .\end{cases}
$$


2. For $v \in(1, \infty)$, we have

$$
\hat{p}_{k}^{v}(t):=\mathbb{P}\left(\hat{M}_{\lambda}^{v}(t)=k\right)= \begin{cases}\mathbb{P}\left(\hat{N}_{\lambda}^{v}(t)=0\right) & \text { if } k=0, \\ \sum_{n=1}^{k} q_{k}^{* n} \mathbb{P}\left(\hat{N}_{\lambda}^{v}(t)=n\right) & \text { if } k \geq 1 .\end{cases}
$$

The aim of this section is to prove that these probability mass functions satisfy suitable versions of Kolmogorov equations with fractional derivatives.

Proposition 1. If $v \in(0,1)$, the probability mass function (10) satisfies the equations

$$
\begin{gathered}
\frac{\mathrm{d}_{\mathrm{C}}^{v}}{\mathrm{~d} t^{\nu}} p_{0}^{\nu}(t)=-\lambda p_{0}^{v}(t), \\
\frac{\mathrm{d}_{\mathrm{C}}^{v}}{\mathrm{~d} t^{\nu}} p_{k}^{v}(t)=-\lambda p_{k}^{v}(t)+\lambda \sum_{i=1}^{k} q_{i} p_{k-i}^{v}(t) \quad \text { for all integers } k \geq 1,
\end{gathered}
$$

with the initial conditions $p_{0}^{v}(0)=1$ and $p_{k}^{v}(0)=0$ for all integers $k \geq 1$.

If $v \in(1, \infty)$, the probability mass function (11) satisfies the equations

$$
\begin{gathered}
\frac{\mathrm{d}_{\mathrm{RL}}^{v}}{\mathrm{~d} t^{\nu}} \hat{p}_{0}^{v}(t)=\lambda \hat{p}_{0}^{v}(t), \\
\frac{\mathrm{d}_{\mathrm{RL}}^{v}}{\mathrm{~d} t^{\nu}} \hat{p}_{k}^{v}(t)=\lambda \hat{p}_{k}^{v}(t)-\lambda \sum_{i=1}^{k} q_{i} \hat{p}_{k-i}^{v}(t) \quad \text { for all integers } k \geq 1,
\end{gathered}
$$

with the initial conditions $\hat{p}_{0}^{v}(0)=1$ and $\hat{p}_{k}^{v}(0)=0$ for all integers $k \geq 1$.

Remarks. For $v=1$, both (12) and (13) reduce to the well-known Kolmogorov forward equations (for instance, we can specialize [10, Chapter 14, Equation (2.3)]). For $k=0$, (12) and (13) coincide with the well-known fractional relaxation equations (see, for example, [2]). For $k>0$, they can be seen as a discrete version of the fractional master equation (see [21, Equation (5.14)] for the case where the common distribution of the jumps have continuous density; see also [8]).

Proof of Proposition 1. In both cases $(v \in(0,1)$ and $v \in(1, \infty))$, the initial conditions trivially hold.

Case $v \in(0,1)$. We have to check that (12) holds. It is known (see e.g. [5, Theorem 2.1]) that

$$
\begin{gathered}
\frac{\mathrm{d}_{\mathrm{C}}^{\nu}}{\mathrm{d} t^{\nu}} \mathbb{P}\left(N_{\lambda}^{\nu}(t)=0\right)=-\lambda \mathbb{P}\left(N_{\lambda}^{\nu}(t)=0\right), \\
\frac{\mathrm{d}_{\mathrm{C}}^{v}}{\mathrm{~d} t^{\nu}} \mathbb{P}\left(N_{\lambda}^{v}(t)=k\right)=-\lambda \mathbb{P}\left(N_{\lambda}^{v}(t)=k\right)+\lambda \mathbb{P}\left(N_{\lambda}^{\nu}(t)=k-1\right) \quad \text { for all integers } k \geq 1,
\end{gathered}
$$

with the initial conditions

$$
\mathbb{P}\left(N_{\lambda}^{v}(0)=0\right)=1, \quad \mathbb{P}\left(N_{\lambda}^{v}(0)=k\right)=0 \quad \text { for all integers } k \geq 1 .
$$

The initial conditions for $\left(\mathbb{P}\left(N_{\lambda}^{v}(0)=k\right)\right)_{k \geq 0}$ meet the ones for $\left(p_{k}^{v}(t)\right)_{k \geq 0}$ in the statement of the proposition. For $k=0$ we have $\left(\mathrm{d}_{\mathrm{C}}^{v} / \mathrm{d} t^{\nu}\right) p_{0}^{\nu}(t)=-\lambda p_{0}^{\nu}(t)$ because $p_{0}^{\nu}(t)=\mathbb{P}\left(N_{\lambda}^{\nu}(t)=0\right)$. 
For $k \geq 1$, we have several steps. Firstly, since

$$
p_{k}^{v}(t)=\sum_{n=1}^{h} q_{k}^{* n} \mathbb{P}\left(N_{\lambda}^{v}(t)=n\right) \quad \text { for all integers } h \geq k,
$$

by (14) we get

$$
\begin{aligned}
\frac{\mathrm{d}_{\mathrm{C}}^{\nu}}{\mathrm{d} t^{\nu}} p_{k}^{v}(t) & =\sum_{n=1}^{k} q_{k}^{* n}\left[-\lambda \mathbb{P}\left(N_{\lambda}^{v}(t)=n\right)+\lambda \mathbb{P}\left(N_{\lambda}^{v}(t)=n-1\right)\right] \\
& =-\lambda \sum_{n=1}^{k} q_{k}^{* n} \mathbb{P}\left(N_{\lambda}^{v}(t)=n\right)+\lambda \sum_{n=1}^{k}\left(\sum_{i=1}^{k} q_{k-i}^{*(n-1)} q_{i}\right) \mathbb{P}\left(N_{\lambda}^{v}(t)=n-1\right) \\
& =-\lambda p_{k}^{v}(t)+\lambda \sum_{i=1}^{k} q_{i} \sum_{n=1}^{k} q_{k-i}^{*(n-1)} \mathbb{P}\left(N_{\lambda}^{v}(t)=n-1\right) .
\end{aligned}
$$

Moreover, since $q_{h}^{* 0}=1_{\{h=0\}}$ and $q_{0}^{* h}=1_{\{h=0\}}$, we get

$$
\begin{aligned}
\sum_{i=1}^{k} q_{i} & \sum_{n=1}^{k} q_{k-i}^{*(n-1)} \mathbb{P}\left(N_{\lambda}^{v}(t)=n-1\right) \\
= & \sum_{i=1}^{k-1} q_{i} \sum_{n=1}^{k} q_{k-i}^{*(n-1)} \mathbb{P}\left(N_{\lambda}^{v}(t)=n-1\right)+q_{k} \sum_{n=1}^{k} q_{0}^{*(n-1)} \mathbb{P}\left(N_{\lambda}^{v}(t)=n-1\right) \\
= & \sum_{i=1}^{k-1} q_{i} \sum_{n=2}^{k} q_{k-i}^{*(n-1)} \mathbb{P}\left(N_{\lambda}^{v}(t)=n-1\right)+q_{k} \mathbb{P}\left(N_{\lambda}^{v}(t)=0\right) \\
= & \sum_{i=1}^{k-1} q_{i} \sum_{j=1}^{k-1} q_{k-i}^{* j} \mathbb{P}\left(N_{\lambda}^{v}(t)=j\right)+q_{k} p_{0}^{v}(t) \\
= & \sum_{i=1}^{k-1} q_{i} p_{k-i}^{v}(t)+q_{k} p_{0}^{v}(t) \\
= & \sum_{i=1}^{k} q_{i} p_{k-i}^{v}(t)
\end{aligned}
$$

this completes the proof, for the first case.

Case $v \in(1, \infty)$. We have to check that (13) holds, and we follow the same structure as in the previous case. The main difference concerns only the initial step, i.e. the analogue of (14) presented above. Thus, we only give some details on how to prove that we have the following equations:

$$
\begin{gathered}
\frac{\mathrm{d}_{\mathrm{RL}}^{v}}{\mathrm{~d} t^{\nu}} \mathbb{P}\left(\hat{N}_{\lambda}^{v}(t)=0\right)=\lambda \mathbb{P}\left(\hat{N}_{\lambda}^{v}(t)=0\right), \\
\frac{\mathrm{d}_{\mathrm{RL}}^{v}}{\mathrm{~d} t^{\nu}} \mathbb{P}\left(\hat{N}_{\lambda}^{v}(t)=k\right)=\lambda \mathbb{P}\left(\hat{N}_{\lambda}^{v}(t)=k\right)-\lambda \mathbb{P}\left(\hat{N}_{\lambda}^{v}(t)=k-1\right) \quad \text { for all integers } k \geq 1,
\end{gathered}
$$


with the initial conditions

$$
\mathbb{P}\left(\hat{N}_{\lambda}^{v}(0)=0\right)=1, \quad \mathbb{P}\left(\hat{N}_{\lambda}^{v}(0)=k\right)=0 \quad \text { for all integers } k \geq 1 .
$$

Thus, since $\hat{N}_{\lambda}^{v}(\cdot)=N_{\lambda}\left(\mathcal{A}^{1 / \nu}(\cdot)\right)$, we have

$$
\mathbb{P}\left(\hat{N}_{\lambda}^{v}(t)=k\right)=\int_{0}^{\infty} \frac{(\lambda x)^{k}}{k !} \mathrm{e}^{-\lambda x} f_{v}(x, t) \mathrm{d} x \quad \text { for all integers } k \geq 0,
$$

where $f_{v}(x, t):=f_{\mathcal{A}^{1 / v}(t)}(x)$ is the density of the random variable $\mathcal{A}^{1 / v}(t)$. Then we get

$$
\begin{aligned}
\frac{\mathrm{d}_{\mathrm{RL}}^{\nu}}{\mathrm{d} t^{\nu}} \mathbb{P}\left(\hat{N}_{\lambda}^{v}(t)=k\right)= & \int_{0}^{\infty} \frac{(\lambda x)^{k}}{k !} \mathrm{e}^{-\lambda x} \frac{\partial}{\partial x} f_{v}(x, t) \mathrm{d} x \\
= & {\left[\frac{(\lambda x)^{k}}{k !} \mathrm{e}^{-\lambda x} f_{v}(x, t)\right]_{x=0}^{x=\infty} } \\
& -\int_{0}^{\infty}\left(-\lambda \mathbb{P}\left(N_{\lambda}(x)=k\right)+\lambda \mathbb{P}\left(N_{\lambda}(x)=k-1\right)\right) f_{v}(x, t) \mathrm{d} x \\
= & \lambda \mathbb{P}\left(\hat{N}_{\lambda}^{v}(t)=k\right)-\lambda \mathbb{P}\left(\hat{N}_{\lambda}^{v}(t)=k-1\right),
\end{aligned}
$$

(the first equality holds by [3, Equations (5.17)-(5.18)] with $\gamma=1 / v$ ); and the proof is complete by taking into account $\mathbb{P}\left(\hat{N}_{\lambda}^{v}(t)=-1\right)=0$ for the case $k=0$.

Remark. If $v \in(1, \infty)$, we can also obtain the alternative equation

$$
\frac{\mathrm{d}}{\mathrm{d} t^{\nu}} \hat{p}_{k}^{v}(t)=-\lambda^{1 / \nu} \sum_{j=0}^{k}(-1)^{j}\left(\begin{array}{c}
1 / \nu \\
j
\end{array}\right) \sum_{l=0}^{\infty} q_{k}^{*(l+j)} \mathbb{P}\left(\hat{N}_{\lambda}^{v}(t)=l\right) \quad \text { for all integers } k \geq 1,
$$

by taking the classical derivative with respect to $t$ in $\hat{p}_{k}^{v}(t)=\sum_{n=1}^{k} q_{k}^{* n} \mathbb{P}\left(\hat{N}_{\lambda}^{v}(t)=n\right)$, and by (6). It is easy to check that, for $v=1$, we meet the classical Kolmogorov equation.

Now, let $N_{1}(\cdot)$ be a nonfractional Poisson process with intensity 1 , independent of $\mathcal{L}^{v}(\cdot)$ (for $v \in(0,1)$ ) and of $\mathscr{A}^{1 / \nu}(\cdot)$ (for $v \in(1, \infty)$ ). Moreover, let $S(\cdot)$ be a subordinator, independent of all the other processes such that the following conditions hold.

1. If $v \in(0,1), M^{v}(t)$ is distributed as $N_{1}\left(S\left(\mathcal{L}^{v}(t)\right)\right)$; in what follows we set $S^{v}(\cdot):=$ $S\left(\mathcal{L}^{v}(\cdot)\right)$, and we have

$$
p_{k}^{\nu}(t)=\mathbb{E}\left[\frac{\left(S^{\nu}(t)\right)^{k}}{k !} \mathrm{e}^{-S^{\nu}(t)}\right]=\mathbb{E}\left[\frac{\left(S\left(\mathcal{L}^{\nu}(t)\right)\right)^{k}}{k !} \mathrm{e}^{-S\left(\mathcal{L}^{\nu}(t)\right)}\right]
$$

for all integers $k \geq 0$.

2. If $v \in(1, \infty), \hat{M}^{v}(t)$ is distributed as $N_{1}\left(S\left(\mathcal{A}^{1 / v}(t)\right)\right)$; in what follows we set $\hat{S}^{v}(\cdot):=$ $S\left(\mathcal{A}^{1 / \nu}(\cdot)\right)$, and we have

$$
\begin{aligned}
\hat{p}_{k}^{v}(t) & =\mathbb{E}\left[\frac{\left(\hat{S}^{v}(t)\right)^{k}}{k !} \mathrm{e}^{-\hat{S}^{\nu}(t)}\right] \\
& =\mathbb{E}\left[\frac{\left(S\left(\mathcal{A}^{1 / v}(t)\right)\right)^{k}}{k !} \mathrm{e}^{-S\left(\mathcal{A}^{1 / v}(t)\right)}\right]
\end{aligned}
$$

for all integers $k \geq 0$. 
In such a case we talk about mixed representation of the fractional versions of the process $M(\cdot)$ in (1). Furthermore, in view of what follows, it is useful to introduce the function $\kappa_{S}$ defined by $\kappa_{S}(\theta):=\log \mathbb{E}\left[\mathrm{e}^{\theta S(1)}\right]$ for all $\theta \in \mathbb{R}$ (note that $\kappa_{S}(\cdot)$ is a nondecreasing function and $\kappa_{S}(0)=0$ ). This function has a strict relationship with the probability generating function $g_{q}$ presented above; actually, by considering standard computations on independent random time-changes for Lévy processes (see e.g. [6]) we can easily check that

$$
\mathbb{E}\left[\mathrm{e}^{\theta S(t)}\right]=\mathrm{e}^{\lambda t\left(g_{q}(1+\theta)-1\right)},
$$

for all $\theta$ such that $1+\theta$ belongs to the domain of convergence of $g_{q}$.

We conclude with the following further formulae. If $v \in(0,1)$, by (3) we have

$$
\mathbb{E}\left[\mathrm{e}^{-\theta S^{\nu}(t)}\right]=E_{\nu, 1}\left(\kappa_{S}(-\theta) t^{\nu}\right) \quad \text { if } \theta \geq 0 .
$$

If $v \in(1, \infty)$, by (2) we have

$$
\mathbb{E}\left[\mathrm{e}^{\theta \hat{S}^{v}(t)}\right]= \begin{cases}\exp \left(-\left(-\kappa_{S}(\theta)\right)^{1 / v} t\right) & \text { if } \theta \leq 0 \\ \infty & \text { if } \theta>0\end{cases}
$$

\subsection{On the concept of overdispersion}

It is well known that a real-valued random variable $Y$ is said to be overdispersed if $\operatorname{var}[Y]-$ $\mathbb{E}[Y]>0$; similarly, for a process $Y(\cdot)$, we have overdispersion if all the random variables $\{Y(t): t>0\}$ are overdispersed. Typically, the compound Poisson process $M(\cdot)$ in (1) exhibits overdispersion when we exclude the trivial case where the random jumps $\left\{X_{n}: n \geq 1\right\}$ are all equal to 1 ; actually, in such a case, we have $M(\cdot)=N_{\lambda}(\cdot)$, i.e. $M(\cdot)$ is a nonfractional Poisson process, and we have $\operatorname{var}[M(t)]-\mathbb{E}[M(t)]=0$, for all $t>0$.

Here we want to study the same feature for the process $M^{v}(\cdot)$ in (7); we do not deal with $\hat{M}^{v}(\cdot)$ in (8) because each random variable $\hat{M}^{v}(t)$ has infinite mean (actually, the moment generating function of $\mathcal{A}^{1 / v}(t)$ is not finite in a neighborhood of the origin; see (2)). It is known (see e.g. [4, Equations (2.7) and (2.8)]) that

$$
\begin{gathered}
\mathbb{E}\left[N_{\lambda}^{v}(t)\right]=\frac{\lambda t^{v}}{\Gamma(v+1)}, \\
\operatorname{var}\left[N_{\lambda}^{v}(t)\right]=\frac{\lambda t^{v}}{\Gamma(v+1)}+\left(\lambda t^{v}\right)^{2} Z(v), \quad \text { where } Z(v):=\frac{1}{v}\left(\frac{1}{\Gamma(2 v)}-\frac{1}{v \Gamma^{2}(v)}\right) .
\end{gathered}
$$

Thus, since we have $Z(v)>0$ for all $v \in(0,1)$ and $Z(1)=0$ (see Figure 1), the fractional Poisson process $N_{\lambda}^{v}(\cdot)$ (for $v \in(0,1)$ ) exhibits overdispersion.

Furthermore, it is known (see e.g. [12, Appendix B.4]) that

$$
\begin{gathered}
\mathbb{E}\left[M^{v}(t)\right]=\mathbb{E}\left[N_{\lambda}^{v}(t)\right] \mathbb{E}\left[X_{1}\right], \\
\operatorname{var}\left[M^{v}(t)\right]=\mathbb{E}\left[N_{\lambda}^{v}(t)\right] \operatorname{var}\left[X_{1}\right]+\operatorname{var}\left[N_{\lambda}^{v}(t)\right] \mathbb{E}^{2}\left[X_{1}\right] ;
\end{gathered}
$$

then, with some computations, we get

$$
\begin{aligned}
\operatorname{var}\left[M^{v}(t)\right]-\mathbb{E}\left[M^{v}(t)\right] & =\mathbb{E}\left[N_{\lambda}^{v}(t)\right]\left(\operatorname{var}\left[X_{1}\right]-\mathbb{E}\left[X_{1}\right]\right)+\operatorname{var}\left[N_{\lambda}^{v}(t)\right] \mathbb{E}^{2}\left[X_{1}\right] \\
& =\frac{\lambda t^{v}}{\Gamma(v+1)}\left(\mathbb{E}\left[X_{1}^{2}\right]-\mathbb{E}\left[X_{1}\right]\right)+\left(\lambda t^{v}\right)^{2} Z(v) \mathbb{E}^{2}\left[X_{1}\right],
\end{aligned}
$$

and $\mathbb{E}\left[X_{1}^{2}\right]-\mathbb{E}\left[X_{1}\right] \geq 0$ because $\mathbb{P}\left(X_{1} \geq 1\right)=1$. In conclusion, the compound process $M^{v}(\cdot)$ in (7) exhibits overdispersion. 


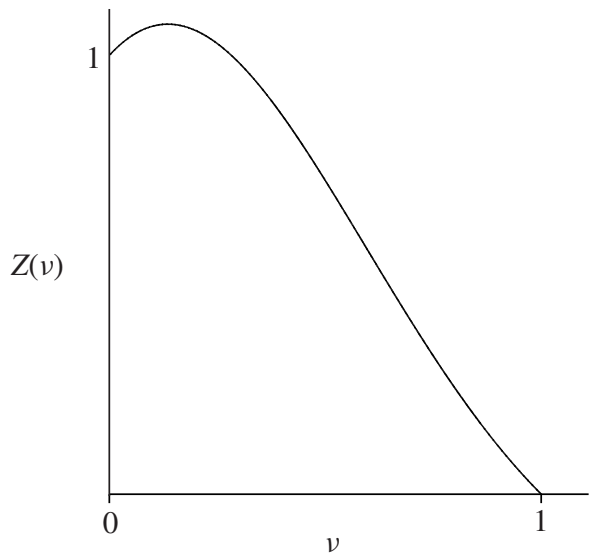

FIGURE 1: Plot of $Z(v):=(1 / v)\left(1 / \Gamma(2 v)-1 / v \Gamma^{2}(v)\right)$ versus $v$.

\section{Examples for the process $M(\cdot)$}

In this section we present some examples for the process $M(\cdot)$ in (1) which allow us to define the corresponding fractional versions $M^{v}(\cdot)$ in $(7)$ and $\hat{M}^{v}(\cdot)$ in (8). More precisely, we consider suitable choices of $\left(q_{k}\right)_{k \geq 1}$ and $\lambda$; moreover, in all cases, the probability mass function $\left(q_{k}\right)_{k \geq 1}$ is a zero truncated negative binomial distribution as in [12, Example B.3.1.5], where $\beta$ in that reference stands for $(1-\alpha) / \alpha$, for some $\alpha \in(0,1)$, and $r>-1$; see Table 1 (where $|u|<1 /(1-\alpha)$ for the probability generating functions). In our examples we have $r=1$ (geometric distribution) for the Pólya-Aeppli process, $r=-\frac{1}{2}$ (extended truncated negative binomial distribution) for the Poisson inverse Gaussian process, and $r=0$ (logarithmic distribution) for the negative binomial process.

Example 1. (Pólya-Aeppli process.) We have

$$
\alpha=1-p \quad \text { and } \quad r=1 \text { for some } p \in(0,1) \text { and } \lambda>0 .
$$

The probability mass functions for the two fractional Pólya-Aeppli processes can be obtained from (4), (5), (10), and (11), with $q_{k}^{* n}=\left(\begin{array}{l}k-1 \\ n-1\end{array}\right)(1-p)^{n} p^{k-n}$ (see e.g. [12, Example 3.19], where $\beta$ in that reference stands for $p /(1-p)$; another interesting reference on this process is [22]).

Alternative formulae for the mixed representation can be obtained from (15) and (16) by considering $S(\cdot)$ as a compound Poisson process with exponentially distributed summands; more precisely, for $\mu=\lambda / p$ and $\beta=(1-p) / p$, we mean $S(\cdot)=\sum_{k=1}^{N_{\mu}(\cdot)} Y_{k}^{(\beta)}$, where $\left\{Y_{k}^{(\beta)}: n \geq 1\right\}$ are i.i.d. random variables with density $f(x)=\beta \mathrm{e}^{-\beta x} 1_{(0, \infty)}(x)$, independent of

TABle 1.

\begin{tabular}{lcc}
\hline & Probability mass function & Probability generating function \\
\hline$r \neq 0$ & $q_{k}=\frac{\left(^{r+k-1}\right)(1-\alpha)^{k}}{\alpha^{-r}-1}$ & $g_{q}(u)=\frac{\alpha^{r}}{1-\alpha^{r}} \frac{1-(1-u(1-\alpha))^{r}}{(1-u(1-\alpha))^{r}}$ \\
$r=0$ (the limit as $r \rightarrow 0)$ & $q_{k}=-\frac{(1-\alpha)^{k}}{k \log \alpha}$ & $g_{q}(u)=\frac{\log (1-u(1-\alpha))}{\log \alpha}$ \\
\hline
\end{tabular}


the (nonfractional) Poisson process $N_{\mu}(\cdot)$ with intensity $\mu$ (see e.g. [9, Section 11.1.2], which concerns a slightly more general situation with the generalized Pólya-Aeppli distribution and the Tweedie distribution; in particular, we recover our case with the Tweedie distribution with parameters $\left.\left(-1, \lambda(1-p) / p^{2},(1-p) / p\right)\right)$.

Now we can state and prove the following result for Example 1 as a consequence of Proposition 1.

Proposition 2. Let us consider the probability mass functions (10) and (11), where $M(\cdot)$ is as in Example 1. If $v \in(0,1)$, we have

$$
\begin{gathered}
\frac{\mathrm{d}_{\mathrm{C}}^{\nu}}{\mathrm{d} t^{\nu}} p_{0}^{\nu}(t)=-\lambda p_{0}^{v}(t), \\
\frac{\mathrm{d}_{\mathrm{C}}^{v}}{\mathrm{~d} t^{\nu}} p_{k}^{v}(t)-(1-\alpha) \frac{\mathrm{d}_{\mathrm{C}}^{\nu}}{\mathrm{d} t^{\nu}} p_{k-1}^{\nu}(t)=-\lambda p_{k}^{v}(t)+\lambda p_{k-1}^{v}(t) \quad \text { for all integers } k \geq 1,
\end{gathered}
$$

with the initial conditions $p_{0}^{v}(0)=1$ and $p_{k}^{v}(0)=0$ for all integers $k \geq 1$.

If $v \in(1, \infty)$, we have

$$
\begin{gathered}
\frac{\mathrm{d}_{\mathrm{RL}}^{\nu}}{\mathrm{d} t^{\nu}} \hat{p}_{0}^{\nu}(t)=\lambda \hat{p}_{0}^{\nu}(t), \\
\frac{\mathrm{d}_{\mathrm{RL}}^{v}}{\mathrm{~d} t^{\nu}} \hat{p}_{k}^{v}(t)-(1-\alpha) \frac{\mathrm{d}_{\mathrm{RL}}^{v}}{\mathrm{~d} t^{\nu}} \hat{p}_{k-1}^{\nu}(t)=\lambda \hat{p}_{k}^{\nu}(t)-\lambda \hat{p}_{k-1}^{v}(t) \quad \text { for all integers } k \geq 1,
\end{gathered}
$$

with the initial conditions $\hat{p}_{0}^{v}(0)=1$ and $\hat{p}_{k}^{v}(0)=0$ for all integers $k \geq 1$.

Remark. The equations (19) and (20) coincide for $v=1$. They are equations for the probability mass functions of the nonfractional Pólya-Aeppli process, and we do not know any references where they appear.

Proof of Proposition 2. Firstly, for $k=0$, we have the same equations as in Proposition 1; thus, we restrict our attention on the case $k \geq 1$. If $v \in(0,1)$, by (12) and by taking into account that $q_{h}=(1-\alpha)^{h-1} \alpha$ (for all integers $h \geq 1$ ), we have

$$
\begin{aligned}
\frac{\mathrm{d}_{\mathrm{C}}^{v}}{\mathrm{~d} t^{v}} p_{k}^{v}(t)-(1-\alpha) \frac{\mathrm{d}_{\mathrm{C}}^{v}}{\mathrm{~d} t^{\nu}} p_{k-1}^{v}(t)= & -\lambda p_{k}^{v}(t)+\lambda \sum_{i=1}^{k}(1-\alpha)^{i-1} \alpha p_{k-i}^{v}(t) \\
& -(1-\alpha)\left(-\lambda p_{k-1}^{v}(t)+\lambda \sum_{i=1}^{k-1}(1-\alpha)^{i-1} \alpha p_{k-1-i}^{v}(t)\right) \\
= & -\lambda p_{k}^{v}(t)+\lambda \alpha p_{k-1}^{v}(t)+\lambda \sum_{i=2}^{k}(1-\alpha)^{i-1} \alpha p_{k-i}^{v}(t) \\
& +\lambda(1-\alpha) p_{k-1}^{v}(t)-\lambda \sum_{i=1}^{k-1}(1-\alpha)^{i} \alpha p_{k-1-i}^{v}(t) \\
= & -\lambda p_{k}^{v}(t)+\lambda p_{k-1}^{v}(t) .
\end{aligned}
$$

If $v \in(1, \infty)$, the proof follows the same lines; we have to consider (13) instead of (12), and there are suitable changes of signs. 
Example 2. (Poisson inverse Gaussian process.) We have

$\alpha=1-\frac{2 \beta}{1+2 \beta}, \quad r=-\frac{1}{2}, \quad \lambda=\lambda_{\beta, \mu}:=\frac{\mu}{\beta}\left((1+2 \beta)^{1 / 2}-1\right) \quad$ for some $\beta, \mu>0$.

We are not aware of any reference with a formula for the convolution densities $q_{k}^{* n}$ as in (9). So (10) and (11) do not give completely explicit formulas for the probability mass functions of the two fractional Poisson inverse Gaussian processes.

Equations (15) and (16) for the mixed representation can be obtained by considering $S(\cdot)$ as an inverse Gaussian process; more precisely, $S(t)=Y_{\mu, \beta}(t)$ should have density

$$
f(x)=\frac{\mu t}{\left(2 \pi \beta x^{3}\right)^{1 / 2}} \exp \left(-\frac{(x-\mu t)^{2}}{2 \beta x}\right) 1_{(0, \infty)}(x) .
$$

Moreover (see e.g. [12, Equation (3.39)]), we have

$$
\mathbb{E}\left[\mathrm{e}^{\theta Y_{\mu, \beta}(t)}\right]= \begin{cases}\exp \left(-\frac{\mu}{\beta} t\left((1-2 \beta \theta)^{1 / 2}-1\right)\right) & \text { if } \theta<\frac{1}{2 \beta}, \\ \infty & \text { if } \theta \geq \frac{1}{2 \beta} .\end{cases}
$$

We remark that the processes $S^{\nu}(\cdot)$ (if $\left.\nu \in(0,1)\right)$ and $\hat{S}^{\nu}(\cdot)$ (if $v \in(1, \infty)$ ) for Example 2 can be seen as fractional inverse Gaussian processes. A different fractional version of this process was defined in [15] by subordinating a fractional Brownian motion to an inverse Gaussian process (in analogy with the so-called fractional Laplace motion; see [13]). Some properties of the process defined in [15] are illustrated in [14].

Example 3. (Negative binomial process.) We have

$$
\alpha=p, \quad r=0, \quad \lambda=-\log p, \quad \text { for some } p \in(0,1) .
$$

The probability mass functions for the two fractional negative binomial processes can be obtained from (4), (5), (10), and (11), with

$$
q_{k}^{* n}=\frac{n !}{(-\log p)^{n}} \frac{(1-p)^{k}|s(k, n)|}{k !},
$$

where $\{|s(k, n)|: k \in\{0,1, \ldots, n\}\}$ are the unsigned Stirling numbers of the first kind (see e.g. [26, Theorem 6]).

Alternative formulae for the mixed representation can be obtained from (15) and (16) by considering $S(\cdot)$ as a Gamma process; more precisely, $S(t)$ should have density

$$
f(x)=\frac{(p /(1-p))^{t}}{\Gamma(t)} x^{t-1} \mathrm{e}^{-(p /(1-p)) x} 1_{(0, \infty)}(x) .
$$

\section{Processes with two fractional parameters}

In this section we generalize the fractional versions of the processes in Examples 1 and 2 by considering a further fractional parameter $\eta \in(0,1)$. Throughout this section we use the notation $N_{1}^{(\eta)}(\cdot)$ for the process $N_{1}^{1 / \eta}(\cdot)$ in (5); then, for each example, we consider independent random time-changes of $N_{1}^{(\eta)}(\cdot)$ in terms of the processes $S^{\nu}(\cdot)$ in (15) and $\hat{S}^{\nu}(\cdot)$ in (16), i.e. the 
fractional versions of the subordinator $S(\cdot)$ which appears in the mixed representation of $M^{v}(\cdot)$ presented in Section 3. Thus, we have

$$
\begin{aligned}
& p_{k}^{\eta, v}(t):=\mathbb{P}\left(M^{\eta, v}(t)=k\right), \quad \text { where } M^{\eta, v}(\cdot)=N_{1}^{(\eta)}\left(S^{\nu}(\cdot)\right) \text { if } v \in(0,1), \\
& \hat{p}_{k}^{\eta, v}(t):=\mathbb{P}\left(\hat{M}^{\eta, v}(t)=k\right), \quad \text { where } \hat{M}^{\eta, v}(\cdot)=N_{1}^{(\eta)}\left(\hat{S}^{\nu}(\cdot)\right) \text { if } v \in(1, \infty) .
\end{aligned}
$$

We remark that we recover the cases presented above by setting $\eta=1$. We shall see that the governing equations for these probability mass functions have not only a fractional time derivative but also the fractional difference operator $(1-B)^{\eta}$ as in (6).

\subsection{Generalized fractional Pólya-Aeppli process}

We start with the following result.

Proposition 3. Let $S(\cdot)=\sum_{k=1}^{N_{\mu}(\cdot)} Y_{k}^{(\beta)}$ be as in Example 1. If $v \in(0,1)$, the probability mass function (22) satisfies

$$
\begin{gathered}
\frac{\mathrm{d}_{\mathrm{C}}^{\nu}}{\mathrm{d} t^{\nu}} p_{0}^{\eta, v}(t)=-\frac{1}{\beta}\left(\mu+\frac{\mathrm{d}_{\mathrm{C}}^{v}}{\mathrm{~d} t^{\nu}}\right) p_{0}^{\eta, v}(t), \\
\frac{\mathrm{d}_{\mathrm{C}}^{v}}{\mathrm{~d} t^{\nu}} p_{k}^{\eta, v}(t)=-\frac{1}{\beta}\left(\mu+\frac{\mathrm{d}_{\mathrm{C}}^{v}}{\mathrm{~d} t^{\nu}}\right)(1-B)^{\eta} p_{k}^{\eta, v}(t) \quad \text { for all integers } k \geq 1,
\end{gathered}
$$

with the initial conditions $p_{0}^{\eta, v}(0)=1$ and $p_{k}^{\eta, v}(0)=0$ for all integers $k \geq 1$.

If $v \in(1, \infty)$, the probability mass function (23) satisfies

$$
\begin{aligned}
& \frac{\mathrm{d}_{\mathrm{RL}}^{v}}{\mathrm{~d} t^{\nu}} \hat{p}_{0}^{\eta, v}(t)=\frac{1}{\beta}\left(\mu-\frac{\mathrm{d}_{\mathrm{RL}}^{v}}{\mathrm{~d} t^{\nu}}\right) \hat{p}_{0}^{\eta, v}(t) \\
& \frac{\mathrm{d}_{\mathrm{RL}}^{\nu}}{\mathrm{d} t^{\nu}} \hat{p}_{k}^{\eta, v}(t)=\frac{1}{\beta}\left(\mu-\frac{\mathrm{d}_{\mathrm{RL}}^{\nu}}{\mathrm{d} t^{\nu}}\right)(1-B)^{\eta} \hat{p}_{k}^{\eta, v}(t) \quad \text { for all integers } k \geq 1 \text {, }
\end{aligned}
$$

with the initial conditions $\hat{p}_{0}^{\eta, v}(0)=1$ and $\hat{p}_{k}^{\eta, v}(0)=0$ for all integers $k \geq 1$.

Remark. The equations (24) and (25) coincide for $v=1$.

Proof of Proposition 3. In both cases $(v \in(0,1)$ and $v \in(1, \infty))$, the initial conditions trivially hold.

Case $v \in(0,1)$. We have

$$
\mathbb{P}\left(S^{v}(t) \in G\right)=\mathbb{P}\left(N_{\mu}\left(\mathcal{L}^{v}(t)\right)=0\right) \mathbf{1}_{G}(0)+\int_{G} f_{v}(x, t) \mathrm{d} x
$$

(for all Borel subsets $G$ of $[0, \infty)$ ), where $f_{v}$ is the density of the absolutely continuous part of the random variable $S^{\nu}(t)$; thus, since $\mathbb{P}\left(N_{\mu}\left(\mathcal{L}^{\nu}(t)\right)=0\right)=E_{\nu, 1}\left(-\mu t^{\nu}\right)$, we obtain

$$
p_{k}^{\eta, v}(t)=E_{v, 1}\left(-\mu t^{\nu}\right) \mathbf{1}_{\{k=0\}}+\int_{0}^{\infty} \mathbb{P}\left(N_{1}^{(\eta)}\left(\mathcal{L}^{v}(z)\right)=k\right) f_{v}(z, t) \mathrm{d} z .
$$

Thus, we get

$$
\begin{aligned}
\frac{\mathrm{d}_{\mathrm{C}}^{\nu}}{\mathrm{d} t^{\nu}} p_{k}^{\eta, v}(t)= & \left(-\mu E_{v, 1}\left(-\mu t^{\nu}\right)+\frac{1}{\beta}\left(\mu+\frac{\mathrm{d}_{\mathrm{C}}^{\nu}}{\mathrm{d} t^{\nu}}\right) f_{\nu}(0, t)\right) \mathbf{1}_{\{k=0\}} \\
& +\frac{1}{\beta}\left(\mu+\frac{\mathrm{d}_{\mathrm{C}}^{\nu}}{\mathrm{d} t^{\nu}}\right) \int_{0}^{\infty} \frac{\mathrm{d}}{\mathrm{d} z}\left\{\mathbb{P}\left(N_{1}^{(\eta)}\left(\mathcal{L}^{\nu}(z)\right)=k\right)\right\} f_{v}(z, t) \mathrm{d} z
\end{aligned}
$$


by [11, Equation (2.4.58)], [3, Equation (2.5)], and an integration by parts (where we take into account that $\left.\mathbb{P}\left(N_{1}^{(\eta)}\left(\mathcal{L}^{v}(0)\right)=k\right)=\mathbf{1}_{\{k=0\}}\right)$. The last equation reduces to

$$
\frac{\mathrm{d}_{\mathrm{C}}^{\nu}}{\mathrm{d} t^{\nu}} p_{k}^{\eta, \nu}(t)=\frac{1}{\beta}\left(\mu+\frac{\mathrm{d}_{\mathrm{C}}^{\nu}}{\mathrm{d} t^{\nu}}\right) \int_{0}^{\infty} \frac{\mathrm{d}}{\mathrm{d} z}\left\{\mathbb{P}\left(N_{1}^{(\eta)}\left(\mathcal{L}^{\nu}(z)\right)=k\right)\right\} f_{v}(z, t) \mathrm{d} z,
$$

because

$$
-\mu E_{v, 1}\left(-\mu t^{\nu}\right)+\frac{1}{\beta}\left(\mu+\frac{\mathrm{d}_{\mathrm{C}}^{\nu}}{\mathrm{d} t^{\nu}}\right) f_{\nu}(0, t)=0 .
$$

We remark that (26) can be checked by inspection after noting that

$$
f_{v}(0, t)=\mu \beta t^{v} E_{v, v+1}^{2}\left(-\mu t^{\nu}\right)=\mu \beta t^{\nu} \sum_{j=0}^{\infty} \frac{(j+1)\left(-\mu t^{\nu}\right)^{j}}{\Gamma(\nu j+v+1)}=-\beta \sum_{j=0}^{\infty} \frac{j\left(-\mu t^{\nu}\right)^{j}}{\Gamma(\nu j+1)}
$$

(by [3, Equation (2.4)] and some computations) and

$$
\frac{\mathrm{d}_{\mathrm{C}}^{\nu}}{\mathrm{d} t^{\nu}} f_{\nu}(0, t)=\mu \beta \sum_{j=0}^{\infty} \frac{(j+1)(-\mu)^{j}\left(\mathrm{~d}_{\mathrm{C}}^{\nu} / \mathrm{d} t^{\nu}\right) t^{\nu j+v}}{\Gamma(v j+v+1)}=\mu \beta \sum_{j=0}^{\infty} \frac{(j+1)\left(-\mu t^{\nu}\right)^{j}}{\Gamma(v j+1)}
$$

(by taking into account that we have $\left(\mathrm{d}_{\mathrm{C}}^{v} / \mathrm{d} t^{v}\right) t^{\nu j+v}=(\Gamma(v j+v+1) / \Gamma(v j+1)) t^{v j}$ by [11, Equation (2.1.17)]).

Finally, we can conclude the proof of this case by using (6) with $\lambda=1$, and with some computations (where we distinguish the cases $k=0$ and $k \geq 1$ ).

Case $v \in(1, \infty)$. We have

$$
\mathbb{P}\left(\hat{S}^{\nu}(t) \in G\right)=\mathbb{P}\left(N_{\mu}\left(\mathcal{A}^{1 / \nu}(t)\right)=0\right) \mathbf{1}_{G}(0)+\int_{G} \hat{f}_{v}(x, t) \mathrm{d} x
$$

(for all Borel subsets $G$ of $[0, \infty)$ ), where $\hat{f}_{\nu}$ is the density of the absolutely continuous part of the random variable $\hat{S}^{v}(t)$; thus, since $\mathbb{P}\left(N_{\mu}\left(\mathcal{A}^{1 / v}(t)\right)=0\right)=\mathrm{e}^{-\mu^{1 / v} t}$, we obtain

$$
\hat{p}_{k}^{\eta, v}(t)=\mathrm{e}^{-\mu^{1 / v} t} \mathbf{1}_{\{k=0\}}+\int_{0}^{\infty} \mathbb{P}\left(N_{1}^{(\eta)}\left(\mathcal{A}^{1 / v}(z)\right)=k\right) \hat{f}_{v}(z, t) \mathrm{d} z .
$$

We follow the same structure as for the proof of the previous case and we give only some details. Firstly, we get

$$
\begin{aligned}
\frac{\mathrm{d}_{\mathrm{RL}}^{\nu}}{\mathrm{d} t^{\nu}} p_{k}^{\eta, v}(t)= & \left(\mu \mathrm{e}^{-\mu^{1 / v} t}-\frac{1}{\beta}\left(\mu-\frac{\mathrm{d}_{\mathrm{RL}}^{v}}{\mathrm{~d} t^{\nu}}\right) f_{v}(0, t)\right) \mathbf{1}_{\{k=0\}} \\
& -\frac{1}{\beta}\left(\mu-\frac{\mathrm{d}_{\mathrm{RL}}^{\nu}}{\mathrm{d} t^{v}}\right) \int_{0}^{\infty} \frac{\mathrm{d}}{\mathrm{d} z}\left\{\mathbb{P}\left(N_{1}^{(\eta)}\left(\mathcal{A}^{1 / \nu}(z)\right)=k\right)\right\} \hat{f}_{\mathcal{v}}(z, t) \mathrm{d} z,
\end{aligned}
$$

by [11, Equation (2.2.15)], [3, Equation (5.8)], and an integration by parts (where we take into account that $\left.\mathbb{P}\left(N_{1}^{(\eta)}\left(\mathcal{A}^{1 / \nu}(0)\right)=k\right)=\mathbf{1}_{\{k=0\}}\right)$. The last equation reduces to

$$
\frac{\mathrm{d}_{\mathrm{RL}}^{\nu}}{\mathrm{d} t^{\nu}} p_{k}^{\eta, \nu}(t)=-\frac{1}{\beta}\left(\mu-\frac{\mathrm{d}_{\mathrm{RL}}^{\nu}}{\mathrm{d} t^{\nu}}\right) \int_{0}^{\infty} \frac{\mathrm{d}}{\mathrm{d} z}\left\{\mathbb{P}\left(N_{1}^{(\eta)}\left(\mathcal{A}^{1 / \nu}(z)\right)=k\right)\right\} \hat{f}_{v}(z, t) \mathrm{d} z,
$$


because

$$
\mu \mathrm{e}^{-\mu^{1 / v} t}-\frac{1}{\beta}\left(\mu-\frac{\mathrm{d}_{\mathrm{RL}}^{\nu}}{\mathrm{d} t^{\nu}}\right) f_{\nu}(0, t)=0 .
$$

We remark that (27) can be checked by inspection after noting that $f_{v}(0, t)=\left(\beta \mu^{1 / v} t / v\right) \mathrm{e}^{-\mu^{1 / v} t}$ (by [3, Equation (5.7)]),

$$
\begin{aligned}
\frac{\mathrm{d}_{\mathrm{RL}}^{v} f_{v}(0, t)}{\mathrm{d} t^{v}} & =\frac{\beta \mu^{1 / v}}{v} \frac{\mathrm{d}_{\mathrm{RL}}^{v}}{\mathrm{~d} t^{v}}\left\{t \mathrm{e}^{-\mu^{1 / v} t}\right\} \\
& =\frac{\beta \mu^{1 / v}}{v} \frac{\mathrm{d}_{\mathrm{RL}}^{v}}{\mathrm{~d} t^{v}}\left(-\frac{\mathrm{d}}{\mathrm{d}\left(\mu^{1 / v}\right)} \mathrm{e}^{-\mu^{1 / v} t}\right) \\
& =-\frac{\beta \mu^{1 / v}}{v} \frac{\mathrm{d}}{\mathrm{d}\left(\mu^{1 / v}\right)} \frac{\mathrm{d}_{\mathrm{RL}}^{v}}{\mathrm{~d} t^{\nu}} \mathrm{e}^{-\mu^{1 / v} t} \\
& =-\frac{\beta \mu^{1 / v}}{v} \frac{\mathrm{d}}{\mathrm{d}\left(\mu^{1 / v}\right)}\left\{\left(\mu^{1 / v}\right)^{v} \mathrm{e}^{-\mu^{1 / v} t}\right\} \\
& =-\frac{\beta \mu^{1 / v}}{v}\left(v \mu \mu^{-1 / v} \mathrm{e}^{-\mu^{1 / v} t}-t \mu \mathrm{e}^{-\mu^{1 / v} t}\right)
\end{aligned}
$$

(by [11, Equation (2.2.15)] (for the equality $\left.\left(\mathrm{d}_{\mathrm{RL}}^{v} / \mathrm{d} t^{\nu}\right) \mathrm{e}^{-\mu^{1 / v} t}=\left(\mu^{1 / v}\right)^{v} \mathrm{e}^{-\mu^{1 / v} t}\right)$, and some computations.)

Finally, we conclude by using (6) with $\lambda=1$ and with some computations (where we distinguish the cases $k=0$ and $k \geq 1$ ).

We already know that, for $\eta=1, M^{\eta, v}(\cdot)$ and $\hat{M}^{\eta, v}(\cdot)$ coincide with the fractional versions (7) and (8) when we deal with the Pólya-Aeppli process $M(\cdot)$ as in Example 1. Then we can check that the results in Proposition 2 can be recovered by considering Proposition 3 with $\eta=1$. Actually, $(1-B)^{1} p_{k}^{1, v}(t)=p_{k}^{1, v}(t)-p_{k-1}^{1, v}(t)$ and, if we rearrange the terms in a suitable way, (24) and (25) with $\eta=1$ meet (19) and (20), respectively, by setting $\mu=\lambda / p=\lambda /(1-\alpha)$ and $\beta=(1-p) / p=\alpha /(1-\alpha)$ as in Example 1 .

\subsection{Generalized fractional Poisson inverse Gaussian process}

From now on we consider the usual symbol $\delta(x)$ for the Dirac delta. Furthermore, we consider the process $S(\cdot)=Y_{\mu, \beta}(\cdot)$ as in Example 2, and we introduce the following notation:

$$
\begin{aligned}
& f_{v}(x, t)=f_{S^{v}(t)}(x) \text { is the density of the random variable } S^{v}(t) \text { for } v \in(0,1), \\
& f_{v}(x, t)=f_{\hat{S}^{v}(t)}(x) \text { is the density of the random variable } \hat{S}^{v}(t) \text { for } v \in(1, \infty)
\end{aligned}
$$

The main result in this subsection is Proposition 5 below, which provides governing equations for the probability mass functions $\left(p_{k}^{\eta, v}(t)\right)_{k \geq 0}$ and $\left(\hat{p}_{k}^{\eta, v}(t)\right)_{k \geq 0}$ when $S(\cdot)$ is as in Example 2 . In its proof we refer to Proposition 4, i.e. a preliminary result for $f_{v}$ and $\hat{f}_{v}$; thus, in some sense, Proposition 4 concerns the fractional versions of the Poisson inverse Gaussian process in Example 2, and not its generalized version considered here.

Proposition 4. Let $f_{v}$ and $\hat{f}_{v}$ be the functions in (28) and (29), respectively. If $v \in(0,1)$, we have

$$
\frac{\partial_{\mathrm{C}}^{v}}{\partial t^{\nu}} \frac{\partial_{\mathrm{C}}^{\nu}}{\partial t^{\nu}} f_{v}(x, t)-2 \frac{\mu}{\beta} \frac{\partial_{\mathrm{C}}^{\nu}}{\partial t^{\nu}} f_{\nu}(x, t)=2 \frac{\mu^{2}}{\beta} \frac{\partial}{\partial x} f_{v}(x, t),
$$

with the initial conditions $f_{v}(x, 0)=\delta(x)$ and $f_{v}(0, t)=0$. 
If $v \in(1, \infty)$, we have

$$
\frac{\partial_{\mathrm{RL}}^{v}}{\partial t^{\nu}} \frac{\partial_{\mathrm{RL}}^{\nu}}{\partial t^{\nu}} \hat{f}_{v}(x, t)+2 \frac{\mu}{\beta} \frac{\partial_{\mathrm{RL}}^{\nu}}{\partial t^{\nu}} \hat{f}_{v}(x, t)=2 \frac{\mu^{2}}{\beta} \frac{\partial}{\partial x} \hat{f}_{v}(x, t),
$$

with the initial conditions $\hat{f}_{v}(x, 0)=\delta(x)$ and $\hat{f}_{v}(0, t)=0$.

Remark. The equations (30) and (31) coincide for $v=1$, and coincide with the equation in [15, Theorem 3.1] (with the same initial conditions).

Proof of Proposition 4. We introduce the following notation: $\tilde{g}(\theta)$, for $\theta \geq 0$, is the Laplace transform of $g(x)$ and $g^{*}(\sigma)$, for $\sigma \geq 0$, is the Laplace transform of $g(t)$ (we need the second one only for $v \in(0,1))$. Furthermore, we recall that, by $(21)$, we have

$$
\kappa_{S}(-\theta)=\log \mathbb{E}\left[\mathrm{e}^{-\theta S(1)}\right]=-\frac{\mu}{\beta}\left((1+2 \beta \theta)^{1 / 2}-1\right) .
$$

Case $v \in(0,1)$. We have $\tilde{f}_{v}(\theta, t)=E_{v, 1}\left(\kappa_{S}(-\theta) t^{\nu}\right)$, by (17), and

$$
\frac{\partial_{\mathrm{C}}^{v}}{\partial t^{\nu}} \tilde{f}_{\nu}(\theta, t)=\kappa_{S}(-\theta) \tilde{f}_{v}(\theta, t)
$$

by [11, Equation (2.4.58)]. Moreover, we have

$$
\left(\frac{\partial_{\mathrm{C}}^{\nu}}{\partial t^{\nu}} g\right)^{*}(\sigma)=\sigma^{\nu} g^{*}(\sigma)-\sigma^{\nu-1} g(0)
$$

by [11, Equation (2.4.63)]. We take the Laplace transforms with respect to $t$ in (32) and, by taking into account $(33)$ with $g(\cdot)=\tilde{f}_{v}(\theta, \cdot)$ for the left-hand side, we get

$$
\sigma^{\nu} \tilde{f}_{v}^{*}(\theta, \sigma)-\sigma^{\nu-1} \underbrace{\tilde{f}_{v}(\theta, 0)}_{=E_{v, 1}(0)=1}=\kappa_{S}(-\theta) \tilde{f}_{v}^{*}(\theta, \sigma) ;
$$

then we have

$$
0=\left(\sigma^{\nu}-\kappa_{S}(-\theta)\right) \tilde{f}_{\nu}^{*}(\theta, \sigma)-\sigma^{\nu-1}=\left(\sigma^{\nu}-\frac{\mu}{\beta}+\frac{\mu}{\beta}(1+2 \beta \theta)^{1 / 2}\right) \tilde{f}_{v}^{*}(\theta, \sigma)-\sigma^{\nu-1}
$$

and, after some computations, we obtain

$$
\left(\sigma^{\nu}\left(\sigma^{\nu}-2 \frac{\mu}{\beta}\right)-2 \frac{\mu^{2}}{\beta} \theta\right) \tilde{f}_{v}^{*}(\theta, \sigma)-\sigma^{\nu-1}\left(\sigma^{\nu}-2 \frac{\mu}{\beta}+\kappa_{S}(-\theta)\right)=0 .
$$

We complete the proof for the case $v \in(0,1)$ by checking that (30) yields (34). This will be done in what follows. We take the Laplace transforms with respect to $x$ in (30) and, after an integration by parts where we take into account that $f_{v}(0, t)=0$, we have

$$
\frac{\partial_{\mathrm{C}}^{\nu}}{\partial t^{\nu}} \frac{\partial_{\mathrm{C}}^{\nu}}{\partial t^{\nu}} \tilde{f}_{v}(\theta, t)-2 \frac{\mu}{\beta} \frac{\partial_{\mathrm{C}}^{\nu}}{\partial t^{\nu}} \tilde{f}_{v}(\theta, t)=2 \frac{\mu^{2}}{\beta} \theta \tilde{f}_{v}(\theta, t) ;
$$

then we take the Laplace transforms with respect to $t$ and, by taking into account (33) with $g(\cdot)=\left(\partial_{\mathrm{C}}^{\nu} / \partial t^{v}\right) \tilde{f}_{v}(\theta, \cdot)$ and with $g(\cdot)=\tilde{f}_{v}(\theta, \cdot)$ together with some computations, we get

$$
\left(\sigma^{\nu}-2 \frac{\mu}{\beta}\right)(\sigma^{\nu} \tilde{f}_{v}^{*}(\theta, \sigma)-\sigma^{\nu-1} \underbrace{\tilde{f}_{v}(\theta, 0)}_{=1})-\left.\sigma^{\nu-1} \frac{\partial_{\mathrm{C}}^{\nu}}{\partial t^{\nu}} \tilde{f}_{v}(\theta, t)\right|_{t=0}=2 \frac{\mu^{2}}{\beta} \theta \tilde{f}_{v}^{*}(\theta, \sigma) .
$$

Finally, we meet (34) because we have $\left.\left(\partial_{\mathrm{C}}^{\nu} / \partial t^{\nu}\right) \tilde{f}_{v}(\theta, t)\right|_{t=0}=\kappa_{S}(-\theta)$ (by (32) with $t=0$ ) and $\tilde{f}_{v}(\theta, 0)=1$. 
Case $v \in(1, \infty)$. We have $\tilde{\hat{f}}_{v}(\theta, t)=\exp \left(-\left(-\kappa_{S}(-\theta)\right)^{1 / v} t\right)$ by $(18)$, and

$$
\begin{gathered}
\frac{\partial_{\mathrm{RL}}^{\nu}}{\partial t^{\nu}} \tilde{\hat{f}}_{v}(\theta, t)=-\kappa_{S}(-\theta) \tilde{\hat{f}}_{\nu}(\theta, t), \\
\frac{\partial_{\mathrm{RL}}^{\nu}}{\partial t^{\nu}} \frac{\partial_{\mathrm{RL}}^{v}}{\partial t^{\nu}} \tilde{\hat{f}}_{v}(\theta, t)=\left(\kappa_{S}(-\theta)\right)^{2} \tilde{\hat{f}}_{\mathcal{v}}(\theta, t)
\end{gathered}
$$

by [11, Equation (2.2.15)]. Then, with some computations we can obtain the equality

$$
\left(\frac{\partial_{\mathrm{RL}}^{v}}{\partial t^{\nu}} \frac{\partial_{\mathrm{RL}}^{v}}{\partial t^{v}} \tilde{\hat{f}}_{v}\right)(\theta, t)+2 \frac{\mu}{\beta}\left(\frac{\partial_{\mathrm{RL}}^{v}}{\partial t^{\nu}} \tilde{\hat{f}}_{v}\right)(\theta, t)=2 \frac{\mu^{2}}{\beta} \theta \tilde{\hat{f}}_{v}(\theta, t) .
$$

We complete the proof by showing that (31) yields (35). We take the Laplace transforms with respect to $x$ in (31), and we have

$$
\left(\frac{\partial_{\mathrm{RL}}^{\nu}}{\partial t^{\nu}} \frac{\partial_{\mathrm{RL}}^{\nu}}{\partial t^{\nu}} \tilde{\hat{f}}\right)(\theta, t)+2 \frac{\mu}{\beta}\left(\frac{\partial_{\mathrm{RL}}^{\nu}}{\partial t^{\nu}} \tilde{\hat{f}}\right)(\theta, t)=\int_{0}^{\infty} \mathrm{e}^{-\theta x} 2 \frac{\mu^{2}}{\beta} \frac{\partial}{\partial x} \hat{f}_{v}(x, t) \mathrm{d} x
$$

then we meet (35) by considering an integration by parts for the right-hand side, where we take into account the equality $\hat{f}_{v}(0, t)=0$.

Now we are ready to state and prove Proposition 5.

Proposition 5. Let $\left(p_{k}^{\eta, v}(t)\right)_{k \geq 0}$ and $\left(\hat{p}_{k}^{\eta, v}(t)\right)_{k \geq 0}$ be the probability mass functions in (22) and (23), respectively, with $S(\cdot)=Y_{\mu, \beta}(\cdot)$ as in Example 2. If $v \in(0,1)$, we have

$$
\frac{\mathrm{d}_{\mathrm{C}}^{\nu}}{\mathrm{d} t^{\nu}} \frac{\mathrm{d}_{\mathrm{C}}^{\nu}}{\mathrm{d} t^{\nu}} p_{k}^{\eta, v}(t)-2 \frac{\mu}{\beta} \frac{\mathrm{d}_{\mathrm{C}}^{\nu}}{\mathrm{d} t^{\nu}} p_{k}^{\eta, v}(t)=2 \frac{\mu^{2}}{\beta}(1-B)^{\eta} p_{k}^{\eta, v}(t) \quad \text { for all integers } k \geq 0,
$$

with the initial conditions $p_{0}^{\eta, v}(0)=1$ and $p_{k}^{\eta, v}(0)=0$ for all integers $k \geq 1$.

If $v \in(1, \infty)$, we have

$$
\frac{\mathrm{d}_{\mathrm{RL}}^{\nu}}{\mathrm{d} t^{\nu}} \frac{\mathrm{d}_{\mathrm{RL}}^{\nu}}{\mathrm{d} t^{\nu}} \hat{p}_{k}^{\eta, v}(t)+2 \frac{\mu}{\beta} \frac{\mathrm{d}_{\mathrm{RL}}^{\nu}}{\mathrm{d} t^{\nu}} \hat{p}_{k}^{\eta, v}(t)=2 \frac{\mu^{2}}{\beta}(1-B)^{\eta} \hat{p}_{k}^{\eta, v}(t) \quad \text { for all integers } k \geq 0,
$$

with the initial conditions $\hat{p}_{0}^{\eta, v}(0)=1$ and $\hat{p}_{k}^{\eta, v}(0)=0$ for all integers $k \geq 1$.

Remark. The equations (36) and (37) coincide for $v=1$.

Proof of Proposition 5. In both cases $(v \in(0,1)$ and $v \in(1, \infty))$, the initial conditions trivially hold.

We start with the case $v \in(0,1)$ and we consider the function $f_{v}$ in (28). Firstly we have

$$
p_{k}^{\eta, v}(t)=\int_{0}^{\infty} \mathbb{P}\left(N_{1}^{(\eta)}(z)=k\right) f_{v}(z, t) \mathrm{d} z \quad \text { for all integers } k \geq 0 .
$$

Then we get

$$
\begin{aligned}
\frac{\mathrm{d}_{\mathrm{C}}^{\nu}}{\mathrm{d} t^{\nu}} \frac{\mathrm{d}_{\mathrm{C}}^{\nu}}{\mathrm{d} t^{\nu}} p_{k}^{\eta, \nu}(t)-2 \frac{\mu}{\beta} \frac{\mathrm{d}_{\mathrm{C}}^{\nu}}{\mathrm{d} t^{\nu}} p_{k}^{\eta, v}(t) \\
\quad=\int_{0}^{\infty} \mathbb{P}\left(N_{1}^{(\eta)}(z)=k\right)\left(\frac{\partial_{\mathrm{C}}^{\nu}}{\partial t^{\nu}} \frac{\partial_{\mathrm{C}}^{\nu}}{\partial t^{\nu}} f_{v}(z, t)-2 \frac{\mu}{\beta} \frac{\partial_{\mathrm{C}}^{\nu}}{\partial t^{\nu}} f_{\nu}(z, t)\right) \mathrm{d} z \\
\quad=\int_{0}^{\infty} \mathbb{P}\left(N_{1}^{(\eta)}(z)=k\right) 2 \frac{\mu^{2}}{\beta} \frac{\partial}{\partial z} f_{v}(z, t) \mathrm{d} z
\end{aligned}
$$


(by (30)); then

$$
\begin{aligned}
\frac{\mathrm{d}_{\mathrm{C}}^{\nu}}{\mathrm{d} t^{v}} \frac{\mathrm{d}_{\mathrm{C}}^{\nu}}{\mathrm{d} t^{\nu}} p_{k}^{\eta, v}(t)-2 \frac{\mu}{\beta} \frac{\mathrm{d}_{\mathrm{C}}^{\nu}}{\mathrm{d} t^{\nu}} p_{k}^{\eta, v}(t) & =-2 \frac{\mu^{2}}{\beta} \int_{0}^{\infty} \frac{\mathrm{d}}{\mathrm{d} z}\left\{\mathbb{P}\left(N_{1}^{(\eta)}(z)=k\right)\right\} f_{v}(z, t) \mathrm{d} z \\
& =2 \frac{\mu^{2}}{\beta}(1-B)^{\eta} \underbrace{\int_{0}^{\infty} \mathbb{P}\left(N_{1}^{(\eta)}(z)=k\right) f_{v}(z, t) \mathrm{d} z}_{=p_{k}^{\eta, v}(t)}
\end{aligned}
$$

(by an integration by parts and by taking into account $f_{v}(0, t)=0$ (for the first equality) and by (6) with $\lambda=1$ (for the second equality)). Thus, (36) holds and this completes the proof for the case $v \in(0,1)$.

The proof for the case $v \in(1, \infty)$ follows the same structure as for the first case; we get (37) by considering a suitable change of sign and, in particular, (31) instead of (30).

We already know that, for $\eta=1, M^{\eta, \nu}(\cdot)$ and $\hat{M}^{\eta, v}(\cdot)$ coincide with the fractional versions, (7) and (8), when we deal with the Poisson inverse Gaussian process $M(\cdot)$ as in Example 2. Then we can check that, for $k=0$, the equations in Proposition 5 can be obtained from the equations in Proposition 1 adapted to this process. Actually, for $v \in(0,1)$, we have

$$
\frac{\mathrm{d}_{\mathrm{C}}^{\nu}}{\mathrm{d} t^{\nu}} p_{0}^{1, \nu}(t)=-\lambda_{\beta, \mu} p_{0}^{1, \nu}(t)=-\frac{\mu}{\beta}\left((1+2 \beta)^{1 / 2}-1\right) p_{0}^{1, \nu}(t)
$$

(by (12)); then we obtain

$$
\frac{\mathrm{d}_{\mathrm{C}}^{\nu}}{\mathrm{d} t^{\nu}} \frac{\mathrm{d}_{\mathrm{C}}^{\nu}}{\mathrm{d} t^{\nu}} p_{0}^{1, \nu}(t)-2 \frac{\mu}{\beta} \frac{\mathrm{d}_{\mathrm{C}}^{\nu}}{\mathrm{d} t^{\nu}} p_{0}^{1, \nu}(t)=2 \frac{\mu^{2}}{\beta} p_{0}^{1, \nu}(t),
$$

with some computations, and this meets (36) in Proposition 5 because $(1-B)^{1} p_{k}^{1, v}(t)=$ $p_{k}^{1, v}(t)-p_{k-1}^{1, v}(t)$ and $p_{-1}^{1, v}(t)=0$ for all $t \geq 0$. For $v \in(1, \infty)$ we have similar computations with suitable changes of signs (we have (13) and (37) in place of (12) and (36), respectively).

\section{Acknowledgements}

We would like to thank an anonymous referee for the careful reading of the paper and for useful suggestions which led to an improved presentation of the paper. We also thank Barbara Pacchiarotti for Figure 1.

\section{References}

[1] Allouba, H. And Zheng, W. (2001). Brownian-time processes: the PDE connection and the half-derivative generator. Ann. Prob. 29, 1780-1795.

[2] Beghin, L. (2012). Fractional relaxation equations and Brownian crossing probabilities of a random boundary. Adv. Appl. Prob. 44, 479-505.

[3] Beghin, L. And Macci, C. (2012). Alternative forms of compound fractional Poisson processes. Abstr. Appl. Anal. 2012, Article ID 747503, 30 pp.

[4] Beghin, L. ANd Orsingher, E. (2009). Fractional Poisson processes and related planar motions. Electron. J. Prob. 14, 1790-1827.

[5] Beghin, L. And Orsingher, E. (2010). Poisson-type processes governed by fractional and higher-order recursive differential equations. Electron. J. Prob. 15, 684-709.

[6] Bertoin, J. (1996). Lévy Processes. Cambridge University Press.

[7] Heyde, C. C. and Leonenko, N. N. (2005). Student processes. Adv. Appl. Prob. 37, 342-365.

[8] Hilfer, R. and Anton, L. (1995). Fractional master equations and fractal time random walks. Phys. Rev. E 51, R848-R851. 
[9] Johnson, N. L., Kemp, A. W. And Kotz, S. (2005). Univariate Discrete Distributions, 3rd edn. John Wiley, Hoboken, NJ.

[10] Karlin, S. and Taylor, H. M. (1981). A Second Course in Stochastic Processes. Academic Press, New York.

[11] Kilbas, A. A., Srivastava, H. M. and Trujillo, J. J. (2006). Theory and Applications of Fractional Differential Equations. Elsevier, Amsterdam.

[12] Klugman, S. A., Panjer, H. H. and Willmot, G. E. (1998). Loss Models. From Data to Decisions. John Wiley, New York.

[13] Kozubowski, T. J., Meerschaert, M. M. and Podgórski, K. (2006). Fractional Laplace motion. Adv. Appl. Prob. 38, 451-464.

[14] Kumar, A. and Vellaisamy, P. (2012). Fractional normal inverse Gaussian process. Methodology Comput. Appl. Prob. 14, 263-283.

[15] Kumar, A., Meerschaert, M. M. and Vellaisamy, P. (2011). Fractional normal inverse Gaussian diffusion. Statist. Prob. Lett. 81, 146-152.

[16] Kumar, A., Nane, E. and Vellaisamy, P. (2011). Time-changed Poisson processes. Statist. Prob. Lett. 81, 1899-1910.

[17] Linde, W. AND SHI, Z. (2004). Evaluating the small deviation probabilities for subordinated Lévy processes. Stoch. Process. Appl. 113, 273-287.

[18] Mainardi, F., Gorenflo, R. and Scalas, E. (2004). A fractional generalization of the Poisson processes. Vietnam J. Math. 32, 53-64.

[19] Mainardi, F., Gorenflo, R. and Vivoli, A. (2005). Renewal processes of Mittag-Leffler and Wright type. Fract. Calc. Appl. Anal. 8, 7-38.

[20] Meerschaert, M. M. And Scheffler, H. P. (2004). Limit theorems for continuous-time random walks with infinite mean waiting times. J. Appl. Prob. 41, 623-638.

[21] Meerschaert, M. M., Nane, E. And Vellaisamy, P. (2011). The fractional Poisson process and the inverse stable subordinator. Electron. J. Prob. 16, 1600-1620.

[22] Minkova, L. D. (2004). The Pólya-Aeppli process and ruin problems. J. Appl. Math. Stoch. Anal. 2004, 221--234.

[23] Orsingher, E. ANd Beghin, L. (2009). Fractional diffusion equations and processes with randomly varying time. Ann. Prob. 37, 206-249.

[24] Orsingher, E. And Polito, F. (2010). Fractional pure birth processes. Bernoulli 16, 858-881.

[25] Orsingher, E. And Polito, F. (2012). The space-fractional Poisson process. Statist. Prob. Lett. 82, 852-858.

[26] Patil, G. P. And WANi, J. K. (1965). On certain structural properties of the logarithmic series distribution and the first type Stirling distribution. Sankhyā A 27, 271-280.

[27] Podlubny, I. (1999). Fractional Differential Equations. Academic Press, San Diego, CA.

[28] Saichev, A. I. AND Zaslavsky, G. M. (1997). Fractional kinetic equations: solutions and applications. Chaos 7, 753-764.

[29] Samorodnitsky, G. and Taqqu, M. S. (1994). Stable Non-Gaussian Random Processes. Chapman \& Hall, New York.

[30] SAto, K. (2001). Subordination and self-decomposability. Statist. Prob. Lett. 54, 317-324.

[31] Scalas, E. (2012). A class of CTRWs: compound fractional Poisson processes. In: Fractional Dynamics, World Scientific, Hackensack, NJ, pp. 353-374. 